\title{
Periodic Solutions of Nonlinear Wave Equations
}

\author{
L. CeSARI \& R. KanNaN
}

\section{Introduction}

In this paper we obtain sufficient conditions for the existence of doubly periodic solutions of the nonlinear wave equation

$$
\begin{gathered}
u_{t s}=f(t, s, u), \\
u(t+T, s)=u(t, s)=u(t, s+T),
\end{gathered}
$$

where $f$ is a given continuous function in $R^{3}$ and $f$ is $T$-periodic in $t$ and $s$.

Periodic solutions of nonlinear hyperbolic partial differential equations have been studied extensively in the recent years $[1,6,7,8,9,10,11,12,13,14]$. The two approaches that have been used are: (i) reduction to an alternative problem and (ii) the Galerkin method and passage to the limit through a sequence of finite dimensional spaces. However in both of these approaches a monotonicity assumption on $f$ with respect to $u$ (e.g., $f_{u} \geqq \gamma \geqq 0$ ) has always been resorted to, this assumption playing a crucial role in both of the above approaches.

In [4] we considered the nonlinear wave equation

$$
u_{t t}-u_{s s}=f\left(t, s, u, u_{t}\right)
$$

together with periodicity conditions on $t$ and Dirichlet-type hypotheses with respect to $s$. We combined both the approaches mentioned above and obtained sufficient conditions for the existence of periodic solutions of the above problem. It must be noted here that, whereas most of the above papers consider problem (3) where $f\left(t, s, u, u_{t}\right)=\varepsilon g\left(t, s, u, u_{t}\right)$ and conclude that for sufficiently small $\varepsilon$ the problem (3) has periodic solutions when $g$ satisfies suitable monotonicity hypotheses, we obtained in [4] quantitative type sufficient conditions which are in the form of a set of inequalities.

We continue here in the same spirit as in [4], obtaining sufficient conditions which are once again expressible as a set of inequalities. However, there are two major differences in this paper. The first is that we study this problem via a direct application of the Schauder fixed point theorem instead of the above approaches. 
This is analogous to the previous results for elliptic problems [5]. Secondly, unlike the above results, we do not require that $f$ satisfy a global monotonicity hypothesis with respect to $u$, but only a local one. We give an example illustrating a general class of nonlinearities $f$ satisfying the hypotheses of our theorem.

\section{Preliminary Remarks Concerning the Linear Problem}

Let $X$ be the Banach space of all functions $u(t, s)$ which are continuous in $R^{2}$ and $T$-periodic in $t$ and $s$, with $\|u\|_{X}=\|u\|_{\infty}=\sup [|u(t, s)|,(t, s) \in G]$, $G=[0, T] \times[0, T]$. For any $u \in X$ let

$$
u^{*}(t, s)=u(t, 0)+u(0, s)-u(0,0)
$$

Then $u^{*}(t, s) \in X$ and also $u^{*}(t, s)=u(t, s)$ on $\partial G$, the boundary of $G$. Further we have the following:

$$
\begin{gathered}
u(t, s)=u^{*}(t, s) \text { on all straight lines } t=h T, s \in \mathbb{R}, \\
\text { and } s=k T, \quad t \in \mathbb{R}, \quad h, k=0, \pm 1, \pm 2, \ldots
\end{gathered}
$$

Any function $u^{*}(t, s)=u_{0}(t)+v_{0}(s)+u_{00}$, where $u_{0}(t)$ and $v_{0}(s)$ are continuous and $T$-periodic in $R$ with $u_{00}$ being a constant, can also be written as

$$
\begin{aligned}
u^{*}(t, s) & =u_{0}(t)+v_{0}(s)+u_{00} \\
& =\left(u^{*}(t, 0)-v_{0}(0)-u_{00}\right)+\left(u^{*}(0, s)-u_{0}(0)-u_{00}\right)+u_{00} \\
& =u^{*}(t, 0)+u^{*}(0, s)-u^{*}(0,0),
\end{aligned}
$$

and thus $u^{*}(t, s)$ is of type (4).

Finally we note that for any $u \in X$, if $u^{*}(t, s)$ is defined by (4), then $u_{1}=$ $u-u^{*}$ is identically zero on $\partial G$ and also on all the lines defined in (5).

We now define $X_{0}$ to be the subspace of all functions $u^{*}$ of $X$ of the form $u^{*}(t, s)=u_{0}(t)+v_{0}(s)+u_{00}$, and let $X_{1}$ be the subspace of $X$ consisting of all functions which are zero on $\partial G$. Let $P: X \rightarrow X_{0}$ be the projection operator defined by $P u=u^{*}$ where $u^{*}$ is defined by (4). By the remarks above, $u-P u$ $=u-u^{*}=u_{1}$ is zero on $\partial G$ and thus $u_{1} \in X_{1}$. Hence every element $u \in X$ is of the form $u=u^{*}+u_{1}$ where $u^{*} \in X_{0}, u_{1} \in X_{1}$, and further

$$
\begin{gathered}
\left|u^{*}(P)\right| \leqq 3 \max [|u(Q)|, Q \in \partial G] \leqq 3\|u\|_{\infty}, \\
\left|u_{1}(P)\right|=\left|u(P)-u^{*}(P)\right| \leqq 4\|u\|_{\infty} .
\end{gathered}
$$

We note that the space of functions $X_{0}$ can also be described differently as follows. Any element $u^{*} \in X_{0}$ is of the form $u_{0}(t)+v_{0}(s)+u_{00}$. Let $\mu_{1}=$ $T^{-1} \int_{0}^{T} u_{0}(t) d t, \quad \mu_{2}=T^{-1} \int_{0}^{T} v_{0}(s) d s$. Then, $u^{*}=u_{0}(t)+v_{0}(s)+u_{00}$ can also be written as

$$
u^{*}=U(t)+V(s)+\mu
$$


where $U(t)=u_{0}(t)-\mu_{1}, \quad V(s)=v_{0}(s)-\mu_{2}$ and $\mu=\mu_{1}+\mu_{2}+u_{00}$. Furthermore, we have $\int_{0}^{T} U(t) d t=\int_{0}^{T} V(s) d s=0$.

For reasons of clarity we now denote by $Y$ the same space $X$ with the same norm. However, we generate the subspaces $Y_{0}$ and $Y_{1}$ differently. Thus for any $F \in Y$ let

$$
\begin{aligned}
m(s) & =T^{-1} \int_{0}^{T} F(t, s) d t-\mu, \\
n(t) & =T^{-1} \int_{0}^{T} F(t, s) d s-\mu, \\
\mu & =T^{-2} \int_{0}^{T} \int_{0}^{T} F(t, s) d s d t, \\
F_{0}(t, s) & =m(s)+n(t)+\mu .
\end{aligned}
$$

Then clearly $T^{-1} \int_{0}^{T} n(t) d t=T^{-1} \int_{0}^{T} m(s) d s=0, n(t)$ and $m(s)$ are continuous in $R$, and $T$-periodic.

Let $Y_{0}$ be the subspace of $Y$ of all continuous functions of the type $U(t)+$ $V(s)+\mu$, where $U(t)$ and $V(s)$ are continuous, $T$-periodic, and of mean value zero. As shown above, for any function $F(t, s) \in Y$ we can define $F_{0}(t, s) \in Y_{0}$ by (6) so that $F_{1}(t, s)=F(t, s)-F_{0}(t, s)$ has mean values zero, i.e., if we define the corresponding $m(s), n(t)$ and $\mu$ as in (6) for $F_{1}$, they will all be zeto. We can now define the projection operator $Q: Y \rightarrow Y_{0}$ by $Q F=F_{0}$, and therefore we can define the subspace $Y_{1}$ of $Y$ by $(I-Q) Y=Y_{1}$. Any $F \in Y$ thus has a unique decomposition $F=F_{0}+F_{1}, F_{0} \in Y_{0}$, and $F_{1} \in Y_{1}$.

We now define the linear operator $H$ on $Y_{1}$ by

$$
v_{1}(t, s)=H F_{1}(t, s)=\int_{0}^{t} \int_{0}^{s} F_{1}(\xi, \eta) d \xi d \eta, \quad F_{1} \in Y_{1} .
$$

Clearly $v_{1}$ is zero on $\partial G$ and on all the lines as in (5). Thus $H: Y_{1} \rightarrow X_{1}$. In other words, for any $F \in Y$,

$$
v_{1}(t, s)=H(I-Q) F=\int_{0}^{t} \int_{0}^{s}[F(\xi, \eta)-m(\eta)-n(\xi)-\mu] d \xi d \eta
$$

is identically zero on $\partial G$ and $v_{1} \in X_{\mathrm{t}}$.

We now show that

$$
\|P\|=3,\|I-P\|=4,\|Q\|=3,\|I-Q\|=4,\|H(I-Q)\|=T^{2} / 4 .
$$

From the above we know already that $\|P\| \leqq 3$ and $\|I-P\| \leqq 4$. For

$$
u^{*}(t, s)=\cos \left(2 \pi T^{-1} t\right)+\cos \left(2 \pi T^{-1} s\right)-1,
$$


we have $\left|u^{*}(t, s)\right| \leqq 1$ on $\partial G$. If $u(t, s)=\left[u^{*}(t, s)\right]_{-1}^{1}$ (that is, $u=u^{*}$ if $-1 \leqq u^{*} \leqq 1, u=+1$ if $u^{*} \geqq 1$, and $u=-1$ if $u^{*} \leqq-1$ ) then, $u=u^{*}$ on $\partial G, u^{*}=P u$, and $|u| \leqq 1$ on $G$. However, for $P_{0}=(T / 2, T / 2)$, we have $u^{*}\left(P_{0}\right)=-3$; hence, $\left\|u^{*}\right\|_{\infty}=3$ while $\|u\|_{\infty}=1$, and $\|P\| \geqq 3$. By comparison we have $\|P\|=3$.

For the same $u$ and $u^{*}$, let

$$
v(t, s)=\left(1-\cos \left(2 \pi T^{-1} t\right)\right)\left(1-\cos \left(2 \pi T^{-1} s\right)\right)
$$

and $\bar{u}(t, s)=[u(t, s)+v(t, s)]_{-1}^{1}$. Now, $v=0$ on $\partial G$, and $u=u=u^{*}$ on $\partial G$, while $u\left(P_{0}\right)=-1, \quad v\left(P_{0}\right)=4, \quad \bar{u}\left(P_{0}\right)=1$, and for $u_{1}=(I-P) \bar{u}=$ $\bar{u}-u^{*}$, we also have $u_{1}\left(P_{0}\right)=1+3=4$. Thus, $\left\|\bar{u}_{1}\right\|_{\infty} \geqq 4,\|\bar{u}\|_{\infty}=1$, and $\|I-P\| \geqq 4$. By comparison we have $\|I-P\|=4$.

Below we denote by $\bar{m}(s), \bar{n}(t)$ the functions defined by $\bar{m}(s)=m(s)+\mu$ and $\bar{n}(t)=n(t)+\mu$, so that $F_{0}=m+n+\mu=\bar{m}+\bar{n}-\mu$, and $\bar{m}, \vec{n}$ are given by the same integrals as $m$ and $n$ in (6) without the term $\mu$. Then, $|\bar{m}| \leqq$ $\|F\|_{\infty}, \quad|\bar{n}| \leqq\|F\|_{\infty}, \quad|\mu| \leqq\|F\|_{\infty}$, and $\left|F_{0}\right|=|\bar{m}+\bar{n}-\mu| \leqq 3\|F\|_{\infty}$. Thus, $\|Q\| \leqq 3$, and $\|I-Q\| \leqq 4$.

Let $(\bar{t}, \bar{s})$ be any point interior to $G$, and let $q, q^{\prime}, q^{\prime \prime}$ denote the sets

$$
\begin{gathered}
q=[(t, s)|| t-\bar{t}|<\delta,| s-\bar{s} \mid<\delta], \\
q^{\prime}=[(t, s)|| t-\bar{t}|<\delta,| s-\bar{s} \mid \geqq \delta, 0 \leqq s \leqq T], \\
q^{\prime \prime}=[(t, s)|| t-\bar{t}|\geqq \delta,| s-\bar{s} \mid<\delta, 0 \leqq t \leqq T],
\end{gathered}
$$

all contained in $G$ for $\delta>0$ sufficiently small. Let $F$ be the discontinuous function defined by $F=-1$ in $q^{\prime} \cup q^{\prime \prime}$, and $F=1$ in $G-\left(q^{\prime} \cup q^{\prime \prime}\right)$; thus, in particular, $F=1$ in $q$. Then, for $\delta$ sufficiently small, we have

$$
\begin{gathered}
\bar{m}(s)=-1+4 T^{-1} \delta \text { for }|s-\bar{s}|<\delta, \\
\bar{n}(t)=-1+4 T^{-1} \delta \text { for }|t-\bar{t}|<\delta, \quad \mu=\left(1-4 T^{-1} \delta\right)^{2},
\end{gathered}
$$

and the function $F_{0}(t, s)=\bar{m}(s)+\bar{n}(t)-\mu$ takes the minimum value

$$
-2+8 T^{-1} \delta-\left(1-4 T^{-1} \delta\right)^{2}
$$

at every point $(t, s) \in q$. This value can be made as close to -3 as we want by taking $\delta$ sufficiently small. Since $F=1$ in $q$, the function $F_{1}=F-F_{0}$ has a value as close to 4 as we want at every point $(t, s) \in q$, again for $\delta>0$ sufficiently small. Now for any fixed such $\delta$ we can replace $F$ by a function $\bar{F}$ continuous in $\mathbb{R}^{2}, T$-periodic in $t$ and $s$, with corresponding $\bar{F}_{0}\left(P_{0}\right)$ as close as we want to -3 , and $\bar{F}_{1}\left(P_{0}\right)$ as close as we want to 4 . Thus, $\|Q\| \geqq 3,\|I-Q\| \geqq 4$, and by comparison $\|Q\|=3,\|I-Q\|=4$. 
We now show that $\|H(I-Q)\| \leqq T^{2} / 4$. Indeed for $F \in Y$, we have

$$
\begin{aligned}
v_{1}(t, s)= & H(I-Q) F=\int_{0}^{t} \int_{0}^{s}\left[F(\xi, \eta)-T^{-1} \int_{0}^{T} F(\xi, \beta) d \beta-T^{-1} \int_{0}^{T} F(\alpha, \eta) d \alpha\right. \\
& \left.+T^{-2} \int_{0}^{T} \int_{0}^{T} F(\alpha, \beta) d \alpha d \beta\right] d \xi d \eta \\
= & \int_{0}^{t} \int_{0}^{s} F(\xi, \eta) d \xi d \eta-\int_{0}^{t} \int_{0}^{T} F(\xi, \eta)(s / T) d \xi d \eta-\int_{0}^{T} \int_{0}^{s} F(\xi, \eta)(t / T) d \xi d \eta \\
& +\int_{0}^{T} \int_{0}^{T} F(\xi, \eta)(t / T)(s / T) d \xi d \eta \\
= & \int_{0}^{T} \int_{0}^{T} F(\xi, \eta) K(t, s ; \xi, \eta) d \xi d \eta,
\end{aligned}
$$

where $K(t, s ; \xi, \eta)=\lambda_{t}(\xi) \lambda_{s}(\eta)$ with $\lambda_{t}(\xi)=1-t / T$ if $0 \leqq \xi \leqq t, \quad \lambda_{t}(\xi)=$ $-t / T$ if $t<\xi \leqq T$, and a similar definition holds for $\lambda_{s}(\eta)$. We note that

$$
\int_{0}^{T}\left|\lambda_{t}(\xi)\right| d \xi=t(1-t / T)+(T-t)(t / T)=2 T^{-1} t(T-t)
$$

Thus $\int_{0}^{T}\left|\lambda_{t}(\xi)\right| d \xi$ attains its maximum value $T / 2$ for $t=T / 2$. Now, for all $t, s \in G$,

$$
\begin{aligned}
\left|v_{1}(t, s)\right| & \leqq \int_{0}^{T} \int_{0}^{T}|F(\xi, \eta)||K(t, s ; \xi, \eta)| d \xi d \eta \\
& \leqq\|F\|_{\infty}\left(\int_{0}^{T}\left|\lambda_{t}(\xi)\right| d \xi\right)\left(\int_{0}^{T}\left|\lambda_{s}(\eta)\right| d \eta\right) \leqq T^{2} / 4\|F\|_{\infty} .
\end{aligned}
$$

Thus $\|H(I-Q)\| \leqq T^{2} / 4$. Now, as before, we consider the discontinuous function $F(t, s)=1$ for $0 \leqq t \leqq T / 2,0 \leqq s \leqq T / 2$ and for $T / 2 \leqq t \leqq T, T / 2 \leqq$ $s \leqq T$ and $F(t, s)=-1$ in the remaining quadrants of $G$. Then $m=n=\mu=0$, $F_{1}=F, \quad v_{1}(t, s)=t s$ for $0 \leqq t \leqq T / 2,0 \leqq s \leqq T / 2$, and analogous expressions hold in the remaining quadrants of $G$. Thus, $v_{1}(T / 2, T / 2)=T^{2} / 4$. Hence $\|H(I-Q)\| \geqq T^{2} / 4$ and thus $\|H(I-Q)\|=T^{2} / 4$.

Finally we observe that $v=H(I-Q) F$ is Lipschitzian. In fact, for $F \in Y$, $|F(t, s)| \leqq M, v(t, s)=H(I-Q) F, \quad$ and $P_{1}=\left(t_{1}, s_{1}\right), \quad P_{2}=\left(t_{2}, s_{1}\right), \quad P_{3}=$ $\left(t_{1}, s_{2}\right) ; P_{4}=\left(t_{2}, s_{2}\right)$ we have

$$
\begin{aligned}
\Delta v=v\left(P_{1}\right)-v\left(P_{2}\right)-v\left(P_{3}\right)+v\left(P_{4}\right) & =\int_{t_{1}}^{t_{2}} \int_{s_{1}}^{s_{2}} F_{1}(\xi, \eta) d \xi d \eta \\
& =\int_{t_{1}}^{t_{2}} \int_{s_{1}}^{s_{2}}[F(\xi, \eta)-m(\eta)-n(\xi)-\mu] d \xi d \eta,
\end{aligned}
$$

and thus $|F| \leqq M,|F-m-n-\mu| \leqq 4 M$, and

$$
|\Delta v|=\left|v\left(P_{1}\right)-v\left(P_{2}\right)-v\left(P_{3}\right)+v\left(P_{4}\right)\right| \leqq 4 M\left|t_{1}-t_{2}\right|\left|s_{1}-s_{2}\right| .
$$


In particular for $s_{1}=s, s_{2}=0,0 \leqq s \leqq T / 2$, we have $v\left(P_{3}\right)=v\left(P_{4}\right)=0$ and thus

$$
\left|v\left(t_{1}, s\right)-v\left(t_{2}, s\right)\right| \leqq 4 M s\left|t_{1}-t_{2}\right| \leqq 2 M T\left|t_{1}-t_{2}\right| \text {. }
$$

Similarly, $\left|v\left(t_{1}, s\right)-v\left(t_{2}, s\right)\right| \leqq 2 M T\left|t_{1}-t_{2}\right|$ for $s_{1}=s, \quad T / 2 \leqq s \leqq T$, $s_{2}=T$. Thus, this relation holds for all $\left(t_{1}, s\right),\left(t_{2}, s\right) \in G$. Analogously, we also have $\left|v\left(t, s_{1}\right)-v\left(t, s_{2}\right)\right| \leqq 2 M T\left|s_{1}-s_{2}\right|$ for all $\left(t, s_{1}\right),\left(t, s_{2}\right) \in G$. Hence, $v=H(I-Q) F$ is Lipschitzian in $\mathbb{R}^{2}$ with Lipschitz constant $2 \sqrt{2} M T$. Also we have

$$
|p|^{-1}|v(t+p, s)-v(t, s)|, \quad|q|^{-1}|v(t, s+q)-v(t, s)| \leqq 2 M T .
$$

We conclude this section by remarking that in the situation described above we have

$$
X=Y, \quad X_{0}=Y_{0}, \quad P \neq Q, \quad I-P \neq I-Q, \quad \text { and } X_{1} \neq Y_{1} .
$$

\section{Existence of Periodic Solutions of the Nonlinear Problem}

We proceed to write the hyperbolic problem (1), (2) as an equivalent pair of operator equations, as in [2]. (For details we refer to [2].) Thus solving (1), (2) is equivalent to finding a fixed point of the transformation $T^{*}$ defined on $X_{1} \times X_{0}$ by $T^{*}:\left(u^{*}, u_{1}\right) \rightarrow\left(\bar{u}^{*}, \bar{u}_{1}\right)$ where

$$
\begin{aligned}
& \bar{u}_{1}=H(I-Q) N\left(u^{*}+u_{1}\right), \\
& \bar{u}^{*}=u^{*}-k S Q N\left(u^{*}+\bar{u}_{1}\right),
\end{aligned}
$$

where $u^{*}, \bar{u}^{*} \in X_{0}$ and $u_{1}, \bar{u}_{1} \in X_{1}, k>0$ is a constant, and $S: Y_{0} \rightarrow X_{0}$ is the identity map. Here we assume that $N u=\phi(t, s)+g(u(t, s))$, with $|\phi(t, s)| \leqq c$, $|g(u)| \leqq C$ for all $(t, s) \in \mathbb{R}^{2}$, and all $u \in \mathbb{R}$ which may be needed.

Let $X_{0}^{*}$ be the set of all elements $u^{*} \in X_{0}$ which are Lipschitzian, i.e., $u^{*}(t, s)$ $=u_{0}(t)+v_{0}(s)+\mu, u_{0}(t), v_{0}(s)$ continuous, $T$-periodic and Lipschitzian, and $\mu$ a constant. For any Lipschitzian function $u(t, s)$ in $R^{2}$ let

$$
\begin{aligned}
& \operatorname{Lip}_{t} u=\sup |p|^{-1}|u(t+p, s)-u(t, s)|, \\
& \operatorname{Lip}_{s} u=\sup |q|^{-1}|u(t, s+q)-u(t, s)| .
\end{aligned}
$$

In $X_{0}^{*}$ we define the norm

$$
\left\|u^{*}\right\|\|=\| u^{*}\left\|_{\infty}+A \max \left[\operatorname{Lip}_{t} u^{*}, \operatorname{Lip}_{s} u^{*}\right]=\right\| u^{*}\left\|_{\infty}+A\right\| u^{*} \|_{*} .
$$

For given $R_{0}, r>0$, let us define the sets

$$
S_{0}^{*}=\left[u^{*} \in X_{0},\left\|u^{*} \mid\right\| \leqq R_{0}\right], S_{1}=\left[u_{1} \in X_{1},\left\|u_{1}\right\|_{\infty} \leqq r\right], \Omega^{*}=S_{0}^{*} \times S_{1} \subset X .
$$

We conclude the proof by assuming first that $T^{*}$ maps $\Omega^{*}$ into $\Omega^{*}$. (In the next section we state the required hypotheses and prove this.) Note that we shall consider $\Omega^{*}$ as a subset of the Banach space $X$ with norm $\|\cdot\|_{\infty}$. We first show that $\Omega^{*}$ is closed in the topology of $X$. Let $u_{n}=u_{n}^{*}+u_{n 1}, n=1,2, \ldots$, be in $\Omega^{*}$ with $u_{n} \rightarrow u$, i.e., $\left\|u_{n}-u\right\|_{\infty} \rightarrow 0$ as $n \rightarrow \infty$. Then, $\left\|u_{n 1}\right\|_{\infty} \leqq r$ and $\left\|u_{n}^{*}\right\|_{\infty}$ 
$+A\left\|u_{n}^{*}\right\|_{*} \leqq R_{0}$. Thus, the elements $u_{n}^{*}$ are uniformly bounded and uniformly Lipschitzian. Hence, $u_{n}^{*} \rightarrow u^{*}$, where $u^{*}$ is Lipschitzian, $\left\|u^{*}\right\|_{*} \leqq \lim \inf \left\|u_{n}^{*}\right\|_{*}$, and $\left\|u^{*}\right\|_{\infty} \leqq \lim \inf \left\|u_{n}^{*}\right\|_{\infty}$. This implies $\left\|u^{*}\right\|_{\infty}+A\left\|u^{*}\right\|_{*} \leqq R_{0}$. Analogously, $u_{n 1} \rightarrow u_{1}$ and $\left\|u_{1}\right\|_{\infty} \leqq \lim \inf \left\|u_{n 1}\right\|_{\infty} \leqq r$. Thus, $\Omega^{*}$ is closed in $X$. It is easily seen that $\Omega^{*}$ is convex.

We now show that $T^{*}: \Omega^{*} \rightarrow \Omega^{*}$ is compact in the topology of $X$. In fact if $u_{n} \in \Omega^{*}, \quad v_{n}=T^{*} u_{n}, \quad$ and $v_{n} \in \Omega^{*}, \quad$ with $u_{n}=u_{n}^{*}+u_{n 1}, \quad v_{n}=v_{n}^{*}+v_{n 1}$, $n=1,2, \ldots$, then $v_{n 1}=H(I-Q) N u_{n}$ is Lipschitzian with constant $2(c+C) T$ while $v_{n}^{*}$ is Lipschitzian with constant $\left\|v_{n}^{*}\right\|_{*} \leqq R_{0} / A$ for all $n$. Also, $\left\|v_{n 1}\right\|_{\infty} \leqq r$ and $\left\|v_{n}^{*}\right\|_{\infty} \leqslant R_{0}$. Hence, by Ascoli's theorem, there is a subsequence $\left[v_{n_{s}}\right]$ which is uniformly convergent to some $v \in X$. Thus, we have shown $T^{*}: \Omega^{*} \rightarrow \Omega^{*}$ is a compact map in the topology of $X$. By Schauder's fixed point theorem $T^{*}$ has at least one fixed point $u$ in $X$, i.e., (1), (2) has a Lipschitz continuous periodic solution.

\section{Hypotheses and Proof of $T^{*}: \Omega^{*} \rightarrow \Omega^{*}$}

As stated above, we assume that $f(t, s, u)=\phi(t, s)+g(u)$ where $\phi$ is continuous and $T$-periodic in $t$ and $s$, and $g$ is continuous and Lipschitzian.

Theorem. Let $\phi: \mathbb{R}^{2} \rightarrow \mathbb{R}$ and $g: \mathbb{R} \rightarrow \mathbb{R}$ satisfy the following: $|\phi(t, s)| \leqq c,|g(u)| \leqq C$ for all $(t, s) \in \mathbb{R}^{2},|u| \leqq R_{0}+r ;$

$g(u) \geqq B$ for $u \geqq b, \quad g(u) \leqq-B$ for $u \leqq-b, \quad g(0)=0 ;$

$|\phi(t, s)-\phi(\xi, s)-\phi(t, \eta)+\phi(\xi, \eta)| \leqq c^{\prime}$ for all $t, s, \xi, \eta ;$

$|\phi(P)-\phi(Q)| \leqq \lambda|P-Q|$ for all $P, Q \in \mathbb{R}^{2}$;

$|g(u)-g(v)| \leqq D|u-v|$ for all $|u|,|v| \leqq R_{0}+r$;

$|g(u)-g(v)| \leqq d|u-v|$ for all $|u|,|v| \leqq R_{0}+r$ and either both

$u, v \geqq b$ or both $u, v \leqq-b$;

and

$$
|u-v-k g(u)+k g(v)| \leqq \gamma|u-v| \text { for all }|u|,|v| \leqq v,
$$

where $b, c, c^{\prime}, d, B, C, D, \gamma, \lambda, v$ are given constants satisfying

$$
0<b<v<R+r_{0}, B<C, 0<d<D, \gamma>0, \lambda>0 \text {. }
$$

Also let $R_{0}, R_{1}, r, 0<R_{1}<R_{0}, r>0$, satisfy

$$
\begin{gathered}
R_{0}-(1+T / A) R_{1}-r \geqq b, \quad R_{0}-R_{1}+r \leqq v,\left(T^{2} / 4\right)(c+C) \leqq r \\
c+c^{\prime}+2(c+C) d T^{2}+2 A(c+C) d T+A \lambda \leqq B-(1+T / A) d R_{1} \\
k\left[C+c+c^{\prime}+2(c+C) d T^{2}+2 A(c+C) d T+A \lambda\right] \\
\leqq 2\left[R_{0}-((1+T / A)+k(T / A) d+k d) R_{1}\right] \\
k c+k c^{\prime}+k D r+(1+4 \gamma)(c+C) T^{2} \\
+2(1+\gamma) A(c+C) T+k A \lambda<(1-\gamma-2 \gamma(T / A)) R_{0} .
\end{gathered}
$$


Then the nonlinear problem (1), (2), i.e.,

$$
u_{t s}=\phi(t, s)+g(u), \quad u(t+T, s)=u(t, s)=u(t, s+T),
$$

has at least one T-periodic solution.

Proof. We first obtain the following auxiliary inequalities by using (9)-(14).

From (12) we derive that, for all $0 \leqq R \leqq R_{1}$,

$$
k\left[c+c^{\prime}+2(c+C) d T^{2}+2 A(c+C) d T+A \lambda\right] \leqq k B-k(1+T / A) d R,
$$

or

$$
\begin{aligned}
R_{0}-k B+k(T / A) d R & +k d R \\
& +k\left[c+c^{\prime}+2(c+C) d T^{2}+2 A(c+C) d T+A \lambda\right] \leqq R_{0} .
\end{aligned}
$$

Thus, by adding and subtracting $R$, and rearranging terms, we also have

$$
\begin{aligned}
{\left[R_{0}-R-k B+k c+\right.} & \left.k c^{\prime}+k(R / A) d T+2 k(c+C) d T^{2}\right] \\
& +(1+k d) R+2 k A(c+C) d T+k A \lambda \leqq R_{0} .
\end{aligned}
$$

In particular

$$
Z_{1}=R_{0}-R-k B+k c+k c^{\prime}+k(R / A) d T+2 k(c+C) d T^{2} \leqq R_{0}
$$

for all $0 \leqq R \leqq R_{1}$. Let

$$
Z_{2}=R_{0}-R-(R / A) T-k C-\left(k c+k c^{\prime}+k(R / A) d T+2 k(c+C) d T^{2}\right) .
$$

If $Z_{2}<0$, letting $Z_{2}^{-}=-Z_{2}$, we have from (13) for $0 \leqq R \leqq R_{1}$,

$$
\begin{aligned}
k\left[C+c+c^{\prime}+2(c+C) d T^{2}+\right. & 2 A(c+C) d T+A \lambda] \\
& \leqq 2 R_{0}-2 R-(T / A) R-k(T / A) d R-k d R
\end{aligned}
$$

or

$$
\begin{aligned}
-R_{0}+R+( & R / A) T+k C+k c+k c^{\prime}+k(R / A) d T \\
& +2 k(c+C) d T^{2}+R(1+k d)+2 k A(c+C) d T+k A \lambda \leqq R_{0},
\end{aligned}
$$

that is

$$
\begin{aligned}
-\left[R_{0}-R-(R / A) T-k C-k c-k c^{\prime}-k(R / A) d T-2 k(c+C) d T^{2}\right] \\
+R(1+k d)+2 k A(c+C) d T+k A \lambda \leqq R_{0} .
\end{aligned}
$$

Thus,

and

$$
Z_{2}^{-}+R(1+k d)+2 k A(c+C) d T+k A \lambda \leqq R_{0}
$$

$$
\begin{array}{r}
R_{0}-R-(R / A) T-k C-k c-k c^{\prime}-k(R / A) d T-2 k(c+C) d T^{2} \\
-R(1+k d)-2 k A(c+C) d T-k A \lambda \geqq-R_{0} .
\end{array}
$$


If $Z_{2}>0$ the above relations are still true since $Z_{2}^{-}=0$ and the term $R(1+k d)+2 k A(c+C) d T+k A \lambda$ already appears in (17) and is, thus, $\leqq R_{0}$.

Finally, from (14) we have, for every $R$ such that $R_{1} \leqq R \leqq R_{0}$,

$$
\begin{aligned}
k c+k c^{\prime}+k D r+(1+4 \gamma)(c+C) T^{2}+2(1+\gamma) A(c+C) T+k A \lambda & \\
& \leqq(1-\gamma) R_{0}-2 \gamma(T / A) R,
\end{aligned}
$$

and

$$
\begin{aligned}
\gamma\left(R_{0}-R\right)+k c+k c^{\prime}+k D r+(1 & +4 \gamma)(c+C) T^{2}+2(1+\gamma) A(c+C) T \\
& +2 \gamma(T / A) R+\gamma R+k A \lambda \leqq R_{0} .
\end{aligned}
$$

In particular

$\gamma\left(R_{0}-R\right)+k c+k c^{\prime}+k D r+(1+4 \gamma)(c+C) T^{2}+2 \gamma(R / A) T \leqq R_{0}$

for all $R_{1} \leqq R \leqq R_{0}$.

As discussed in the previous section, the proof is complete if $T^{*}$ maps $\Omega^{*}$ into itself. Recall that $T^{*}$ is given by $T^{*}:\left(u^{*}, u_{1}\right) \rightarrow\left(\bar{u}^{*}, \bar{u}_{1}\right)$ where

$$
\bar{u}_{1}=H(I-Q) N\left(u^{*}+u_{1}\right), \quad \bar{u}^{*}=u^{*}-k \operatorname{SQN}\left(u^{*}+\bar{u}_{1}\right) .
$$

Now

$$
\|N u\|_{\infty}=\|\phi(t, s)+g(u(t, s))\|_{\infty} \leqq c+C .
$$

Also $\|m(s)\|_{\infty} \leqq 2(c+C),\|n(t)\|_{\infty} \leqq 2(c+C),|\mu| \leqq c+C,\|m(s)+n(t)+\mu\|_{\infty}$ $\leqq 3(c+C)$, and $\|(I-Q) N u\|_{\infty} \leqq 4(c+C)$. From the hypotheses (9) and (11) we have

$$
\left.\left\|\bar{u}_{1}\right\|_{\infty}=\left\|K_{1} u\right\|_{\infty}=\|H(I-Q) N u\|_{\infty} \leqq\left(T^{2} / 4\right)\right)(c+C) \leqq r
$$

for all $u=u^{*}+u_{1} \in \Omega^{*}$. Thus $K_{1}: \Omega^{*} \rightarrow S_{1}$ where $K_{1} u=H(I-Q) N u$. Also by (8) we have

$$
\operatorname{Lip}_{t} \bar{u}_{1} \leqq 2(c+C) T, \quad \operatorname{Lip}_{s} \bar{u}_{1} \leqq 2(c+C) T .
$$

Before we discuss the map $u^{*} \rightarrow \bar{u}^{*}$ we consider the expression $u^{*}-Q N u$. Given any function $u \in X$ and points $P_{1}=(t, s), P_{2}=(\xi, s), \quad P_{3}=(t, \eta)$ and $P_{4}=(\xi, \eta)$ in $R^{2}$, let $\Delta u=u\left(P_{1}\right)-u\left(P_{2}\right)-u\left(P_{3}\right)+u\left(P_{4}\right)$. Let $f(t, s, u)$ be any function which is continuous in $R^{3}$ and $T$-periodic in $t$ and $s$, and let $N u=f(t, s, u(t, s))$ for $u \in X$, so that $N: X \rightarrow Y$. For $u \in X, u=u^{*}+u_{1}$, $u^{*} \in X_{0}, u_{1} \in X_{1}$, we define $\psi \in X$ by

$$
\psi(t, s)=u^{*}(t, s)+H(I-Q) N u
$$

$$
=u^{*}(t, s)+\int_{0}^{t} \int_{0}^{s}[f(\xi, \eta, u(\xi, \eta))-m(\eta)-n(\xi)-\mu] d \xi d \eta,
$$


where $m, \eta, \mu$ are the mean values of $F(\xi, \eta)=f(\xi, \eta, u(\xi, \eta))$; thus $P \psi=u^{*}$, $(I-P) \psi=H(I-Q) N u$, and $F_{0}(t, s)=Q N u=m(s)+n(t)+\mu$. Then

$$
\begin{aligned}
Q N u & =m(s)+n(t)+\mu \\
& =T^{-1} \int_{0}^{T} F_{0}(\xi, s) d \xi+T^{-1} \int_{0}^{T} F_{0}(t, \eta) d \eta-T^{-2} \int_{0}^{T} \int_{0}^{T} F_{0}(\xi, \eta) d \xi d \eta \\
& =T^{-2} \int_{0}^{T} \int_{0}^{T}[f(\xi, s, u(\xi, s))+f(t, \eta, u(t, \eta))-f(\xi, \eta, u(\xi, \eta))] d \xi d \eta .
\end{aligned}
$$

Thus

$$
\begin{aligned}
u^{*}-k S Q N u= & u^{*}(t, s)-k S(Q N u)(t, s) \\
= & u^{*}(t, s)-k T^{-2} \int_{0}^{T} \int_{0}^{T}\left[f\left(\xi, s, u^{*}(\xi, s)+u_{1}(\xi, s)\right)\right. \\
& \left.+f\left(t, \eta, u^{*}(t, \eta)+u_{1}(t, \eta)\right)-f\left(\xi, \eta, u^{*}(\xi, \eta)+u_{1}(\xi, \eta)\right)\right] d \xi d \eta \\
= & {\left[u^{*}(t, s)-k f\left(t, s, u^{*}(t, s)\right)\right] } \\
& +k\left[f\left(t, s, u^{*}(t, s)\right)-f\left(t, s, u^{*}(t, s)+u_{1}(t, s)\right)\right] \\
& +k T^{-2} \int_{0}^{T} \int_{0}^{T}\left[f\left(t, s, u^{*}(t, s)+u_{1}(t, s)\right)\right. \\
& \quad-f\left(\xi, s, u^{*}(\xi, s)+u_{1}(\xi, s)\right)-f\left(t, \eta, u^{*}(t, \eta)+u_{1}(t, \eta)\right) \\
& \left.+f\left(\xi, \eta, u^{*}(\xi, \eta)+u_{1}(\xi, \eta)\right)\right] d \xi d \eta .
\end{aligned}
$$

Note that in the second equation defining the map $T^{*}$ we have taken $\bar{u}_{1}$ instead of $u_{1}$.

Two cases arise in the consideration of the map $u \rightarrow \bar{u}^{*}$.

Case 1. $u=u^{*}+u_{1} \in \Omega^{*},\left\|u^{*}\right\|_{\infty}=R_{0}-R, \operatorname{Lip}_{t} u^{*}, \operatorname{Lip}_{s} u^{*} \leqq R / A$ for some $R, 0 \leqq R \leqq R_{1}<R_{0}$. Note that

$$
\left\|u^{*}\right\|=\left\|u^{*}\right\|_{\infty}+A \operatorname{Max}\left(\operatorname{Lip}_{t} u^{*}, \operatorname{Lip}_{s} u^{*}\right) \leqq R_{0}-R+A(R / A)=R_{0}
$$

with $\left\|u_{1}\right\|_{\infty} \leqq r,\left\|\bar{u}_{1}\right\|_{\infty} \leqq r$.

Observe also that

$$
\left\|u^{*}\right\|_{*}=\left\|u_{0}(t)+v_{0}(s)+u_{00}\right\|_{*} \leqq R / A,
$$

so that $u_{0}(t)$ can have at most an oscillation $\leqq(R / A)(T / 2)$ in $0 \leqq t \leqq T$ since $u_{0}(0)=u_{0}(T)$. Analogously, $v_{0}(s)$ can have at most an oscillation $\leqq(R / A)(T / 2)$ in $0 \leqq s \leqq T$ since $v_{0}(0)=v_{0}(T)$. Thus, $u^{*}$ has the same value at the four corners of $[0, T] \times[0, T]$, and $u^{*}$ has an oscillation $\leqq(R / A) T$ in $[0, T] \times[0, T]$. Thus,

$$
\begin{gathered}
\left|u^{*}(t, s)\right| \geqq\left\|u^{*}\right\|_{\infty}-(R / A) T=R_{0}-R-(R / A) T \text { for all }(t, s), \\
\left|u^{*}(t, s)+\bar{u}_{1}(t, s)\right| \geqq\left|u^{*}(t, s)\right|-\left|\bar{u}_{1}(t, s)\right| \geqq R_{0}-R-(R / A) T-r \geqq b
\end{gathered}
$$

for all $(t, s)$. 
Thus, either $u^{*}(t, s)+\bar{u}_{1}(t, s) \geqq b$ for all $(t, s)$, or $u^{*}(t, s)+\bar{u}_{1}(t, s) \leqq-b$ for all $(t, s)$.

If $u^{*}(t, s)+\bar{u}_{1}(t, s) \geqq b$ for all $(t, s)$, then $g\left(u^{*}(t, s)+\bar{u}_{1}(t, s)\right) \geqq B$ for all $(t, s)$.

We consider next the expression (21) with $f=\phi(t, s)+g(u)$, where the integration over $[0, T] \times[0, T]$ can be performed instead over $[t-T / 2, t+T / 2]$ $\times[s-T / 2, s+T / 2]$ because of the double $T$-periodicity of the integrand.

The expression under integration, or $\Delta f$, can now be estimated by noting that $\Delta f=\Delta \phi+\Delta g,|\Delta \phi| \leqq c^{\prime}$, that $g$ is Lipschitzian of constant $D$ in $\left[-R_{0}-r\right.$, $\left.R_{0}+r\right]$ and of constant $d$ outside $(-b, b)$, that $u^{*}$ is Lipschitzian of constant $R / A$, that $\bar{u}_{1}$ is Lipschitzian of constant $2(c+C) T$ in each variable (from relation (8)), and finally that any two corresponding points below are at a distance $\leqq T / 2:$ thus

$$
\begin{aligned}
|\Delta f| \leqq & |\Delta \phi|+\left|g\left(u^{*}(t, s)+\bar{u}_{1}(t, s)\right)-g\left(u^{*}(\xi, s)+\bar{u}_{1}(\xi, s)\right)\right| \\
& +\left|g\left(u^{*}(t, \eta)+\bar{u}_{1}(t, \eta)\right)-g\left(u^{*}(\xi, \eta)+\bar{u}_{1}(\xi, \eta)\right)\right| \\
\leqq & c^{\prime}+d[(R / A)+2(c+C) T](T / 2)+d[(R / A)+2(c+C) T](T / 2) \\
= & c^{\prime}+d T(R / A)+2(c+C) d T^{2} .
\end{aligned}
$$

From (21) and the relations above we have now, by force of (16) and (18),

$$
\begin{gathered}
\bar{u}^{*}(t, s)=\left[u^{*}(t, s)-k g\left(u^{*}(t, s)+\bar{u}_{1}(t, s)\right)\right]-k \phi(t, s) \\
+k T^{-2} \int_{t-T / 2}^{t+T / 2} \int_{s-T / 2}^{s+T / 2}(\Delta \phi+\Delta g) d \xi d \eta, \\
\bar{u}^{*}(t, s) \leqq R_{0}-R-k B+k c+k c^{\prime}+k(R / A) d T+2 k(c+C) d T^{2} \\
=Z_{1}=R_{0}-R(1-k d(T / A))-k B+\left(k c+k c^{\prime}+2 k(c+C) d T^{2}\right) \leqq R_{0},
\end{gathered}
$$

$$
\begin{aligned}
\bar{u}^{*}(t, s) \geqq R_{0} & -R-(R / A) T-k C \\
& -\left(k c+k c^{\prime}+k(R / A) d T+2 k(c+C) d T^{2}\right)=Z_{2} \geqq-R_{0} .
\end{aligned}
$$

If $u^{*}(t, s)+\bar{u}_{1}(t, s) \leqq-b$ for all $(t, s)$, then $g\left(u^{*}(t, s)+\bar{u}_{1}(t, s)\right) \leqq-B$ for all $(t, s)$, and as above $-R_{0} \leqq \bar{u}^{*}(t, s) \leqq R_{0}$ for all $(t, s)$.

Concerning the Lipschitz constant of $\bar{u}^{*}$, note that

$$
\begin{aligned}
\bar{u}^{*}(t, s)=u^{*}(t, s)-k(m(s) & +n(t)+\mu)=-k(m(s)+\mu)+u^{*}(t, s) \\
& -k T^{-1} \int_{0}^{T} g\left(u^{*}(t, \eta)+\bar{u}_{1}(t, \eta)\right) d \eta-k T^{-1} \int_{0}^{T} \phi(t, \eta) d \eta .
\end{aligned}
$$


Thus

$$
\begin{aligned}
& \bar{u}^{*}(t+p, s)-\bar{u}^{*}(t, s)=u^{*}(t+p, s)-u^{*}(t, s) \\
& -k T^{-1} \int_{0}^{T}[\phi(t+p, \eta)-\phi(t, \eta)] d \eta \\
& \quad-k T^{-1} \int_{0}^{T}\left[g\left(u^{*}(t+p, \eta)+\bar{u}_{1}(t+p, \eta)\right)-g\left(u^{*}(t, \eta)+\bar{u}_{1}(t, \eta)\right)\right] d \eta .
\end{aligned}
$$

Hence

$$
\begin{aligned}
&\left|\bar{u}^{*}(t+p, s)-\bar{u}^{*}(t, s)\right| \leqq \\
&\left.+k T^{-1} \int_{0}^{T} d \mid A\right)|p|+k \lambda|p| \\
& \quad \leqq(R / A)|p|+k d((R / A)+2(c+C) T)|p|+k \lambda|p| .
\end{aligned}
$$

Thus $\operatorname{Lip}_{t} \bar{u}^{*} \leqq(R / A)(1+k d)+2 k(c+C) d T+k \lambda$.

The same inequality holds for $\operatorname{Lip}_{s} \bar{u}^{*}$. Thus, by (16), (17), (22) and (23), $\left\|\mid \bar{u}^{*}\right\|\|=\| \bar{u}^{*} \|_{\infty}+A \max \left(\operatorname{Lip}_{t} \bar{u}^{*}, \operatorname{Lip}_{s} \bar{u}^{*}\right)$

$$
\leqq \max \left(Z_{1}, Z_{2}^{-}\right)+R(1+k d)+2 k A(c+C) d T+k A \lambda \leqq R_{0} .
$$

In other words, we require here that for all $0 \leqq R \leqq R_{1}$ both the following relations hold:

$$
\begin{gathered}
{\left[R_{0}-R-k B+k c+k c^{\prime}+k(R / A) d T+2 k(c+C) d T^{2}\right]} \\
+R(1+k d)+2 k A(c+C) d T+k A \lambda \leqq R_{0}, \\
{\left[R_{0}-R-(R / A) T-k C-k c-k c^{\prime}-k(R / A) d T-2 k(c+C) d T^{2}\right]^{-}} \\
+R(1+k d)+2 k A(c+C) d T+k A \lambda \leqq R_{0}
\end{gathered}
$$

and these are exactly (15) and (17) respectively.

Case 2. $u=u^{*}+u_{1} \in \Omega^{*},\left\|u^{*}\right\|_{\infty}=R_{0}-R,\left\|u^{*}\right\|_{*} \leqq R / A$, for some $R$, $0<R_{1} \leqq R \leqq R_{0} . \quad$ Again, $\quad\left\|u^{*}\right\|\left\|\leqq R_{0}, \quad\right\| u_{1}\left\|_{\infty} \leqq r, \quad\right\| \bar{u}_{1}\left\|_{\infty} \leqq r, \quad\right\| \bar{u}_{1} \|_{*} \leqq$ $2(c+C) T$. Then,

$$
\left|u^{*}(t, s)\right| \leqq R_{0}-R \leqq R_{0}-R_{1} \leqq \nu,\left|u^{*}(t, s)+\bar{u}_{1}(t, s)\right| \leqq R_{0}-R+r \leqq \nu,
$$

and by (9) and (10) with $v=0$, also $\left|u^{*}-k g\left(u^{*}\right)\right| \leqq \gamma\left|u^{*}\right|$. Hence

$$
\left|u^{*}(t, s)-k g\left(u^{*}(t, s)\right)-k \phi(t, s)\right| \leqq k c+\gamma\left|u^{*}(t, s)\right| \leqq k c+\gamma\left(R_{0}-R\right) .
$$

By (9) we have also

$$
\left|g\left(u^{*}(t, s)\right)-g\left(u^{*}(t, s)+\bar{u}_{1}(t, s)\right)\right| \leqq D\left|\bar{u}_{1}(t, s)\right| \leqq D r .
$$


For $P_{1}=(t, s), P_{2}=(\xi, s), P_{3}=(t, \eta), P_{4}=(\xi, \eta)$, we have for the expression $\Delta g$ with $u(P)=u^{*}(P)+\bar{u}_{1}(P)$,

$$
\begin{aligned}
k|\Delta g|= & \left|k g\left(u\left(P_{1}\right)\right)-k g\left(u\left(P_{2}\right)\right)-k g\left(u\left(P_{3}\right)\right)+k g\left(u\left(P_{4}\right)\right)\right| \\
& =\mid\left[u^{*}\left(P_{1}\right)-u^{*}\left(P_{2}\right)-u^{*}\left(P_{3}\right)+u^{*}\left(P_{4}\right)\right] \\
& +\left[\bar{u}_{1}\left(P_{1}\right)-\bar{u}_{1}\left(P_{2}\right)-\bar{u}_{1}\left(P_{3}\right)+\bar{u}_{1}\left(P_{4}\right)\right] \\
& -\left[u\left(P_{1}\right)-u\left(P_{2}\right)-k g\left(u\left(P_{1}\right)\right)+k g\left(u\left(P_{2}\right)\right)\right] \\
& +\left[u\left(P_{3}\right)-u\left(P_{4}\right)-k g u\left(P_{3}\right)+k g\left(u\left(P_{4}\right)\right)\right] \mid .
\end{aligned}
$$

The first bracket, or $\Delta u^{*}$, is identically zero; indeed since $u^{*}=u_{00}+u_{0}(t)+$ $v_{0}(s)$, we have

$$
\begin{array}{r}
\Delta u^{*}=\left(u_{00}+u_{0}(t)+v_{0}(s)\right)-\left(u_{00}+u_{0}(\xi)+v_{0}(s)\right)-\left(u_{00}+u_{0}(t)+v_{0}(\eta)\right) \\
+\left(u_{00}+u_{0}(\xi)+v_{0}(\eta)\right)=0 .
\end{array}
$$

The second bracket in (24), or $\Delta \bar{u}_{1}$, is estimated in $\S 2$ by $\left|\Delta \bar{u}_{1}\right| \leqq 4(c+C)$ $|t-\xi||s-\eta|$. If the integration in (21) is performed on the square $[t-T / 2$, $t+T / 2] \times[s-T / 2, s+T / 2], \quad$ then $|t-\xi| \leqq T / 2, \quad|s-\eta| \leqq T / 2$, and the contribution of $\Delta \bar{u}_{1}$ in (21) is $\leqq(c+C) T^{2}$.

Let us denote the third and fourth brackets in (24) by $\Sigma_{P_{1} P_{2}}$ and $\Sigma_{P_{3} P_{4}}$ respectively. By using relations (10) we have

$$
\begin{aligned}
\left|\Sigma_{P_{1} P_{2}}\right| \leqq \gamma\left|u\left(P_{1}\right)-u\left(P_{2}\right)\right| & \leqq \gamma[(R / A)+2(c+C) T]\left|P_{1}-P_{2}\right| \\
& \leqq \gamma(R / A) T+2 \gamma(c+C) T^{2},
\end{aligned}
$$

and an analogous estimate holds for $\Sigma_{P_{3} P_{4}}$. Thus,

$$
\begin{gathered}
|\Delta g| \leqq 0+\left|\Delta \bar{u}_{1}\right|+2 \gamma(R / A) T+4 \gamma(c+C) T^{2} \\
\left|\bar{u}^{*}(t, s)\right| \leqq \gamma\left(R_{0}-R\right)+k c+k D r+0+(c+C) T^{2} \\
+2 \gamma(R / A) T+4 \gamma(c+C) T^{2}+k c^{\prime}
\end{gathered}
$$

and by (20) we have $\left\|\bar{u}^{*}\right\|_{\infty} \leqq R_{0}$. Concerning the Lipschitz constant of $\bar{u}^{*}$, we shall need estimates different from those for case 1 . First we have

$$
\begin{gathered}
\bar{u}^{*}=u^{*}-k S Q N\left(u^{*}+u_{1}\right), \\
\bar{u}^{*}(t, s)=u^{*}(t, s)-k[m(s)+n(t)+\mu] \\
=-k(m(s)+\mu)+u^{*}(t, s)-k T^{-1} \int_{0}^{T}\left[\phi(t, \eta)+g\left(u^{*}(t, \eta)+\bar{u}_{1}(t, \eta)\right)\right] d \eta \\
=-k(m(s)+\mu)-k T^{-1} \int_{0}^{T} \phi(t, \eta) d \eta-\bar{u}_{1}(t, s) \\
+T^{-1} \int_{0}^{T}\left[u^{*}(t, s)+\bar{u}_{1}(t, s)-k g\left(u^{*}(t, \eta)+\bar{u}_{1}(t, \eta)\right)\right] d \eta .
\end{gathered}
$$


Finally, we have for $\bar{u}^{*}(t, s)$ the following expression:

$$
\begin{aligned}
\bar{u}^{*}(t, s)= & -k(m(s)+\mu)-k T^{-1} \int_{0}^{T} \phi(t, \eta) d \eta-\bar{u}_{1}(t, s) \\
& +T^{-1} \int_{0}^{T}\left[u^{*}(t, s)+\bar{u}_{1}(t, s)-u^{*}(t, \eta)-\bar{u}_{1}(t, \eta)\right] d \eta \\
& +T^{-1} \int_{0}^{T}\left[u^{*}(t, \eta)+\bar{u}_{1}(t, \eta)-k g\left(u^{*}(t, \eta)+\bar{u}_{1}(t, \eta)\right)\right] d \eta .
\end{aligned}
$$

For $\bar{u}^{*}(t+p, s)$ we shall the analogous expression with $t+p$ replacing $t$, and so by subtraction

$$
\begin{aligned}
\bar{u}^{*}(t+p, s) & -\bar{u}^{*}(t, s)=-k T^{-1} \int_{0}^{T}[\phi(t+p, \eta)-\phi(t, \eta)] d \eta \\
& -\left[\bar{u}_{1}(t+p, s)-\bar{u}_{1}(t, s)\right] \\
& +T^{-1} \int_{0}^{T}\left[u^{*}(t+p, \eta)+\bar{u}_{1}(t+p, \eta)-u^{*}(t, \eta)-\bar{u}_{1}(t, \eta)\right. \\
& \left.-k g\left(u^{*}(t+p, \eta)+\bar{u}_{1}(t+p, \eta)\right)+k g\left(u^{*}(t, \eta)+\bar{u}_{1}(t, \eta)\right)\right] d \eta \\
& +T^{-1} \int_{0}^{T}\left[u^{*}(t+p, s)-u^{*}(t+p, \eta)-u^{*}(t, s)+u^{*}(t, \eta)\right] d \eta \\
& +T^{-1} \int_{0}^{T}\left[\bar{u}_{1}(t+p, s)-\bar{u}_{1}(t, s)-\bar{u}_{1}(t+p, \eta)+\bar{u}_{1}(t, \eta)\right] d \eta .
\end{aligned}
$$

The fifth term in the second member is zero since the integrand is equal to

$$
\begin{aligned}
u_{0}(t+p)+v_{0}(s)+u_{00}-u_{0}(t+p)-v_{0}(\eta)-u_{00} & -u_{0}(t)-v_{0}(s)-u_{00} \\
& +u_{0}(t)+v_{0}(\eta)+u_{00}=0 .
\end{aligned}
$$

The second term cancels the first part of the sixth term. Here

$$
\begin{gathered}
\left|u^{*}(t, \eta)+u_{1}(t, \eta)\right| \leqq R_{0}-R+r \leqq R_{0}-R_{1}+r \leqq \nu, \\
\left|u^{*}(t+p, \eta)+u_{1}(t+p, \eta)\right| \leqq v,
\end{gathered}
$$

and by force of (9) and (10)

$$
\begin{aligned}
&\left|\bar{u}^{*}(t+p, s)-\bar{u}^{*}(t, s)\right| \leqq k T^{-1} \int_{0}^{T}|\phi(t+p, \eta)-\phi(t, \eta)| d \eta \\
& \quad+\gamma T^{-1} \int_{0}^{T}\left|u^{*}(t+p, \eta)-u^{*}(t, \eta)+\bar{u}_{1}(t+p, \eta)-\bar{u}_{1}(t, \eta)\right| d \eta \\
& \quad+T^{-1} \int_{0}^{T}\left|\bar{u}_{1}(t+p, \eta)-\bar{u}_{1}(t, \eta)\right| d \eta \\
& \leqq k \lambda|p|+\gamma((R / A)+2(c+C) T)|p|+2(c+C) T|p| \\
&=(k \lambda+\gamma R / A+2(1+\gamma)(c+C) T)|p| .
\end{aligned}
$$


Thus $\operatorname{Lip}_{t} \bar{u}^{*} \leqq k \lambda+\gamma R / A+2(1+\gamma)(c+C) T$. The same estimate holds for $\operatorname{Lip}_{s} \bar{u}^{*}$. Finally, by force of (14) and (25), also

$$
\begin{gathered}
\|\| \bar{u}^{*}\|=\| \bar{u}^{*} \|_{\infty}+A \max \left(\operatorname{Lip}_{t} \bar{u}^{*}, \operatorname{Lip}_{s} \bar{u}^{*}\right) \\
\leqq \gamma\left(R_{0}-R\right)+k c+k D r+(1+4 \gamma)(c+C) T^{2} \\
+2 \gamma(R / A) T+k c^{\prime}+k A \lambda+\gamma R+2(1+\gamma) A(c+C) T \\
\leqq \gamma(1+2 T / A) R_{0}+\left(k c+k c^{\prime}+k A \lambda+k D r+(1+4 \gamma)(c+C) T^{2}\right. \\
+2(1+\gamma) A(c+C) T) \\
\leqq \gamma(1+2 T / A) R_{0}+(1-\gamma-2 \gamma T / A) R_{0}=R_{0} .
\end{gathered}
$$

Thus we have proved that $T^{*}: Q^{*} \rightarrow Q^{*}$. The existence of a periodic solution of (1), (2) now follows from Section 3.

Remark. The estimate

$$
\left|\phi-T^{-2} \int_{0}^{T} \int_{0}^{T} \Delta \phi d \xi d \eta\right| \leqq|\phi|+|\Delta \phi| \leqq c+c^{\prime}
$$

has been used above. This estimate is particularly useful when $\phi \in \operatorname{ker} E$ and thus $\Delta \phi \equiv 0, c^{\prime}=0$. In general $|\Delta \phi| \leqq c^{\prime} \leqq 4 c$ and $c+c^{\prime}+5 c$, but this estimate is too generous. Since the first of the four terms in $\Delta \phi$ cancels the term $\phi$ outside the integral, it is enough to replace $c+c^{\prime}$ by $3 c$ in relations (11), (12) and (13).

\section{Example}

To begin with, let us consider the problem

$$
u_{t s}=\phi(t, s)+g(u), \quad u(t+T, s)=u(t, s)=u(t, s+T),
$$

with

$$
\begin{gathered}
g(-u)=g(u), g(u)=\arctan u \text { for } 0 \leqq u \leqq v=1.3984=\tan (0.95), \\
g(u)=0.95+\varrho \sin \sigma(u-v) \text { for } u \geqq v
\end{gathered}
$$

where $\varrho, \sigma$ are arbitrary constants with $|\varrho| \leqq 0.06,|\varrho \sigma| \leqq 0.4$.

Here we have $|g(u)| \leqq 0.95+0.06=1.01$ for all $u$, and we can take $C=$ 1.01. We also have

$$
\begin{gathered}
1 \geqq g^{\prime}(u)=\left(1+u^{2}\right)^{-1} \geqq\left(1+v^{2}\right)^{-1}=0.33834 \text { for } 0 \leqq u \leqq v=1.3984, \\
\left|g^{\prime}(u)\right|=|\varrho \sigma \cos \sigma(u-v)| \leqq|\varrho \sigma| \leqq 0.4 \text { for } u \geqq v .
\end{gathered}
$$

Thus, we can take $D=1$. For $b=1.2346=\tan (0.89)$, and $b \leqq u \leqq v$, we have $g(u) \geqq 0.89$; for $u \geqq v$ we have $g(u) \geqq 0.95-0.06=0.89$. Thus, we can take $B=0.89$. 
Again, for $b \leqq u \leqq v$, we have

$$
0.33834=\left(1+v^{2}\right)^{-1} \leqq g^{\prime}(u) \leqq\left(1+b^{2}\right)^{-1}=0.3961592,
$$

and for the function $g$, continuous in $(-\infty,+\infty)$, we have

$$
|g(u)-g(v)| \leqq d|u-v| \text { for all } u, v \geqq b \text {, and } d=0.4 \text {. }
$$

Finally, for $k=1$, we have $|u-v-g(u)-g(v)| \leqq \gamma|u-v|$, or

$$
\left|1-(u-v)^{-1}(g(u)-g(v))\right| \leqq \gamma \text { for all } 0 \leqq u, v \leqq \nu
$$

with $\gamma=1-\left(1+v^{2}\right)^{-1}=0.66166$. Let us take $A=1, R_{0}=3, R_{1}=1.61$, $r=0.01, T=10^{-1}$. It remains to verify relations (11)-(14). Indeed,

$$
\begin{gathered}
R_{0}-(1+T / A) R_{1}-r=3-(1.1)(1.61)-0.01=1.219<1.2346=b . \\
R_{0}-R_{1}+r=3-1.61+0.01=1.4=v, \\
\left(T^{2} / 4\right)(c+C)=(0.0025)(c+1.01)=(0.0015) c+0.002525<0.01=r,
\end{gathered}
$$

or $c<2.99$. The remaining relations become

$$
\begin{aligned}
c+c^{\prime}+2 & (c+C) d T^{2}+2 A(c+C) d T+A \lambda+(1+T / A) d R_{1} \\
= & c+c^{\prime}+2(c+1.01)(0.4) 10^{-2}+2(c+1.01)(0.4) 10^{-1} \\
& +\lambda+(1.1)(0.4)(1.61) \\
= & (1.088) c+c^{\prime}+\lambda+0.79<0.89=B, \\
C+c+c^{\prime}+ & +c+C) d T^{2}+2 A(c+C) d T+A \lambda+2(1+T / A) R_{1} \\
& +k(T / A) d R_{1}+k d R_{1} \\
= & 1.01+c+c^{\prime}+2(c+1.01)(0.4) 10^{-2}+2(c+1.01)(0.4) 10^{-1}+\lambda \\
& +2(1.1)(1.61)+(0.1)(0.4)(1.61)+(0.4)(1.61) \\
= & (1.088) c+c^{\prime}+\lambda+5.34928<6=2 R_{0}, \\
+ & +(1+4 \gamma)(c+C) T^{2}+2(1+\gamma) A(c+C) T+\lambda A \\
= & c+c^{\prime}+0.01+3.64664(c+1.01) 10^{-2} \\
& +2(1.66166)(c+1.01) 10^{-1}+\lambda \\
= & (1.369) c+c^{\prime}+\lambda+0.38249<0.61803 \\
= & \left(1-0.66166-2(0.66166) 10^{-1}\right) 3=(1-\gamma-2 \gamma T / A) R_{0} .
\end{aligned}
$$

Thus, all we have to verify is that

$$
\text { (1.088) } c+c^{\prime}+\lambda<0.1 \text { and (1.369) } c+c^{\prime}+\lambda<0.23554 \text {. }
$$

For instance, for

$$
\phi(t, s)=10^{-3} \sin \left(2 \pi T^{-1} t\right)
$$


we have $c=10^{-3}, \phi \in \operatorname{ker} E, \Delta \phi \equiv 0, c^{\prime}=0, \phi_{t}=10^{-3}\left(2 \pi T^{-1}\right) \cos \left(2 \pi^{-1} t\right)$, $\lambda=10^{-3}(62.8319)=(0.062832)$, and

$$
\begin{gathered}
(1.088) c+c^{\prime}+\lambda=0.06392<0.1, \\
(1.369) c+c^{\prime}+\lambda=0.064201<0.23554 .
\end{gathered}
$$

Similarly, for

$$
\phi(t, s)=10^{-3} \sin \left(2 \pi T^{-1}\right)(t+s)
$$

we have

$$
\Delta=10^{-3} \cdot 4 \sin \left(2^{-1}(t-\eta)\right) \sin \left(2^{-1}(t+s+\xi+\eta)\right) \sin \left(2^{-1}(t-\xi)\right) .
$$

Hence, $c=10^{-3}, c^{\prime}=4 \cdot 10^{-3}, \lambda=0.064172$, and

$$
\begin{gathered}
1.088 c+c^{\prime}+\lambda=0.067920<0.1, \\
(1.369) c+c^{\prime}+\lambda=0.068201<0.23554 .
\end{gathered}
$$

The solutions whose existence we have just verified of course satisfy

$$
|u(t, s)| \leqq R_{0}+r=3+0.01=3.01 .
$$

Thus, both equations

$$
u_{t s}=10^{-3} \sin (2 \pi)(10 t)+g(u)
$$

and

$$
u_{t s}=10^{-3} \sin (2 \pi)(10)(t+s)+g(u),
$$

with $g(u)$ defined by (26), have periodic solutions of period $T=0.1$ with $|u(t, s)| \leqq 3.01$.

Note. Research partially supported by U.S. Army Research Grant DAAG 29-80-C0060 at the University of Texas at Arlington and by U.S.AFOSR Research Grant 71-2122 at the University of Michigan.

\section{References}

1. L. CESARI, Existence in the large of periodic solutions of hyperbolic partial differential equations. Archive Rational Mech. Anal. 20, 170-190 (1965).

2. L. CESARI, Functional analysis, nonlinear differential equations, and the alternative method. Nonlinear Functional Analysis and Differential Equations (L. CesarI, R. Kannan, J. D. Schuur, eds.). M. Dekker, New York, 1976, 1-197.

3. L. CeSARI \& R. KANNAN, Existence of solutions of nonlinear hyperbolic equations. Annali Scuola Normale Sup. Pisa (4) 6, 573-592 (1979) and 7, p. 715 (1980).

4. L. CeSARI \& R. KANNAN, Solutions of hyperbolic differential equations at resonance. J. Nonlinear Analysis 6, 751-805 (1982).

5. L. Cesari \& R. Kannan, An abstract existence theorem at resonance. Proc. Amer. Math. Soc. 63, 221-225 (1977).

6. J. K. HALE, Periodic solutions of a class of hyperbolic equations. Archive Rational Mech. Anal. 23, 380-398 (1967). 
7. W. S. Hall, Periodic solutions of a class of weakly nonlinear evolution equations. Archive Rational Mech. Anal. 39, 294-322 (1970).

8. M. NAKAO, Bounded, periodic and almost periodic classical solutions of some nonlinear wave equations with a dissipative term. J. Math. Soc. Japan 30, 375-394 (1978).

9. H. Petzeltova, Periodic solutions of the equation $u_{t t}+u_{x x x x}=f(., ., u)$. Czechoslovak Math. J. 23, 269-285 (98) (1973).

10. G. Prodi, Soluzioni periodiche dell'equazione delle onde con termine dissipativo non lineare. Rend. Sem. Padova, (1965), 37-49.

11. G. Prouse, Soluzioni periodiche dell'equazione delle onde non omogenea con termine dissipativo quadratico. Ricerche di Mat. 13, 261-280 (1964).

12. P. H. Rabinowitz, Periodic solutions of nonlinear hyperbolic partial differential equations. Comm. Pure Appl. Math. 20, 145-205 (1967).

13. P. H. Rabinowitz, Free vibrations for a semilinear wave equation. Comm. Pure Appl. Math. 31, 31-68 (1978).

14. O. VeJVODA, Periodic solutions of a linear and weakly nonlinear wave equation in one dimension, I. Czech. Math. J. 14, 341-382 (1964).

Department of Mathematics

University of Michigan

Ann Arbor, Michigan 48109

and

Department of Mathematics

University of Texas at Arlington

Arlington, Texas 76019

(Received September 1, 1980) 